\title{
Characterisation of ESKAPE Pathogens with Special Reference to Multidrug Resistance and Biofilm Production in a Nepalese Hospital
}

\author{
Rosy Pandey (D) ${ }^{1,2}$ \\ Shyam Kumar Mishra iD 3,4 \\ Angela Shrestha' \\ 'St. Xavier's College (Tribhuvan \\ University), Department of Microbiology, \\ Kathmandu, Nepal; ${ }^{2}$ National Public \\ Health Laboratory, Kathmandu, Nepal; \\ ${ }^{3}$ UNSW Sydney, School of Optometry \\ and Vision Science, Sydney, Australia; \\ ${ }^{4}$ Tribhuvan University, Institute of \\ Medicine, Maharajgunj Medical Campus, \\ Department of Microbiology, Kathmandu, \\ Nepal
}

Background: "ESKAPE" is an acronym for a group of life-threatening nosocomial pathogens, viz, Enterococcus faecium, Staphylococcus aureus, Klebsiella pneumoniae, Acinetobacter baumannii, Pseudomonas aeruginosa and Enterobacter spp. Global efforts on controlling multidrug-resistant (MDR) organisms have been hampered by their ability to escape antibacterial drugs. This study was undertaken to determine the prevalence of ESKAPE pathogens with prime focus on biofilm production and antibiotic resistance.

Methods: A total of 8756 clinical samples were processed for the isolation and identification of ESKAPE pathogens following standard microbiological procedures. These isolates were subjected to antimicrobial sensitivity test as per Clinical and Laboratory Standards Institute (CLSI) guidelines. Test for MDR, extended-spectrum $\beta$-lactamase (ESBL), metallo$\beta$-lactamase (MBL), methicillin-resistant Staphylococcus aureus (MRSA), vancomycinresistant Enterococcus (VRE) was done by the disk diffusion and E-test methods. In the case of VRE molecular detection was done for vanA and vanB genes. All the isolates were processed for biofilm detection by the tube adherence method.

Results: The percentage distribution of Enterococcus faecium was 5.5\%, S. aureus $33.4 \%$, K. pneumoniae $33.0 \%$, A. baumannii $8.6 \%$, P. aeruginosa $18.6 \%$, and Enterobacter aerogenes $0.9 \%$. MRSA was $57.6 \%$, and vancomycin resistance among Enterococcus faecium was $20 \%$. ESBL- and MBL-producing K. pneumoniae were $16.1 \%$, and $8.1 \%$, A. baumannii $10.3 \%$ each and $P$. aeruginosa $10.7 \%$ and $8.3 \%$, respectively. A total of $42.3 \%$ of isolates were biofilm producers. Linezolid was the drug of choice for VRE. Ampicillin-sulbactam was most useful against $A$. baumannii apart from polymyxins, whereas piperacillintazobactam was effective against other Gram-negative bacteria. VanA gene was detected in all the VRE isolates.

Conclusion: This study estimates the burden of the ESKAPE organisms and their antimicrobial resistance pattern in a hospital setting. A high percentage of drug resistance and biofilm production was noted; hence antimicrobial resistance surveillance targeting ESKAPE pathogens should be incorporated in the infection control policy in Nepal.

Keywords: extended-spectrum $\beta$-lactamase; ESBL, ESKAPE pathogens, metallo- $\beta$ lactamase; MBL, methicillin-resistant Staphylococcus aureus; MRSA, vancomycin-resistant Enterococcus; VRE

\section{Background}

The emergence of bacterial pathogens with acquired resistance to almost all available antimicrobials is one of the major concerns today. ${ }^{1}$ These pathogens are also named "superbugs," particularly Enterococcus faecium, Staphylococcus aureus,
St. Xavier's College, Post Box: 7437,

Maitighar, Kathmandu, 4460I, Nepal

Tel +977-9803005426

Email roseypandey@gmail.com 
Klebsiella pneumoniae, Acinetobacter baumannii, Pseudomonas aeruginosa, and Enterobacter spp (ESKAPE). The inefficiency of antimicrobials against these pathogens is due to various resistance mechanisms such as drug inactivation, modification of drug binding sites/targets, changes in cell permeability and/or mutation. $^{2}$

Methicillin-resistant Staphylococcus aureus (MRSA) emerged in the 1960s and was first identified in 1961 in the United Kingdom. ${ }^{3}$ Its nosocomial infection rate in the United States and the United Kingdom was around $60-70 \%$ in $2004 .{ }^{4}$ However, the incidence of MRSA bloodstream infections in the United States dropped off $74 \%$ and $40 \%$, respectively, in the hospitals and communities from 2005 to $2016 .{ }^{5}$ In Nepal, the prevalence of MRSA in the hospital setting was found as high as $68 \%$ while it was around $50 \%$ in the community; a similar study showed that $42 \%$ of $S$. aureus isolates were MRSA in a tertiary care teaching hospital. ${ }^{6,7}$

The production of enzymes, that irreversibly inactivate $\beta$-lactam antimicrobials, e.g., extended-spectrum $\beta$ lactamase (ESBL) and carbapenemase like metallo- $\beta$ lactamase (MBL), has contributed to the success of Gramnegative members of ESKAPE pathogens in the clinical settings. ${ }^{8}$ Carbapenems are often referred to as last resort antibiotics that are used for the treatment of multidrugresistant (MDR) Gram-negative bacterial infections as they possess activity against ESBL-producing organisms too. ${ }^{8}$ However, Gram-negative members of ESKAPE pathogens producing MBLs are resistant to carbapenems, thus posing urgent threat in clinical settings.

On several occasions, ESKAPE clinical isolates are MDR, extensively drug-resistant (XDR), or pandrugresistant (PDR). The misuse of antimicrobials and the proneness of organisms to carry the resistant genes conferring MDR infection is a major issue these days. On the same ground, drug-resistant strains of Staphylococcus aureus, mainly MRSA, vancomycin-resistant Enterococcus (VRE) and $\beta$-lactam resistant pathogens have proven to be the leading threats in the clinical arena. ${ }^{9}$ These pathogens can survive in the hospital setting for a longer period of time, escape the biocidal effect easily and can be transported from one individual to other, hence spreading in the community and hospital. ${ }^{10}$ Acinetobacter baumannii isolates harboring NDM-1 and 16S rRNA methylase ArmA have been found to have disseminated in medical settings in Nepal. ${ }^{11}$ Therefore, there is an outcry for more clinically potent antibiotics or novel antimicrobials including antimicrobial peptides and their mimics. ${ }^{12}$

Biofilm is a layer of microbially-derived cell clusters embedded in a matrix of extracellular polysaccharide, called polysaccharide intercellular adhesins (PIA). It has shown that ESKAPE pathogens are potential biofilm producers which make them resistant to antimicrobials by creating a layer hindering antimicrobial penetration. ${ }^{13}$

In Nepal, studies focusing on the collective evaluation of prevalence and antimicrobial resistance profile of ESKAPE pathogens is lacking. Therefore, in this study, status of ESKAPE pathogens, antimicrobial ineffectiveness due to production of biofilms or enzymes like $\beta$ lactamases, methicillin resistance among Staphylococcus aureus, vancomycin resistance among Enterococcus faecium have been addressed. Among the nine phenotypic variants of vancomycin resistance in Enterococci (vanA, vanB, vanC, vanD, vanE, vanG, vanL, vanM and vanN), strains possessing vanA and $v a n B$ are found to be responsible for human infections. ${ }^{14}$ Therefore, in this study, our focus was to detect $\operatorname{van} A / \operatorname{van} B$ gene(s) on the clinical isolates of VRE.

\section{Methods}

This was a cross-sectional descriptive study conducted from February to July 2018 at a 100-bed hospital in the capital city of Nepal. A total of 8756 specimens (urine, swabs and bodily fluids - pus, blood, sputum, tracheal aspirate, ear swab, wound swab, throat swab, pleural fluid, endotracheal secretion, cerebrospinal fluid and semen) were processed aseptically from medical, surgical and intensive care units of inpatient department (IPD). Clinical and microbiological details were recorded of each patient. A repeated specimen from the same patient within 48 hours was not included in the study to exclude selection bias.

\section{Sample Collection and Processing}

The clinical specimens were collected from February to July 2018 and they were processed following standard protocol of the American Society for Microbiology (ASM) and analyzed accordingly. ${ }^{15}$ Antimicrobial susceptibility test (AST) was done for all the clinical isolates as per the guidelines of Clinical and Laboratory Standards Institute (CLSI). ${ }^{16}$ Wet mount microscopy was done for urine specimens and Gram's staining was done on all the other bodily fluids, respiratory aspirates and swabs. For blood sample processing, BD BACTEC FX40 was 
employed with Standard Aerobic or Peds Plus medium. Urine specimens were cultured in Cystine Lactose Electrolyte Deficient (CLED) medium by the semiquantitative standard loop method, and for other specimens, Chocolate agar (CA), 5\% Blood agar (BA) and MacConkey agar (MA) were used. The CA plates were incubated in a $\mathrm{CO}_{2}$ enriched environment $\left(5-10 \% \mathrm{CO}_{2}\right)$ at $37^{\circ} \mathrm{C}$ for 24 hours. The BA, MA, and CLED plates were incubated at $37^{\circ} \mathrm{C}$ for 24 hours in an aerobic atmosphere. The identification of significant isolates was done following standard microbiological techniques. ${ }^{15}$ Composite Quality scoring was done considering the relative ratio of polymorphs and epithelial cells on the samples to rule out bacterial colonization from infection for lower respiratory tract specimens.

\section{Bacterial Identification}

Identification of bacteria was done based on standard microbiological techniques which involved Gram's staining, morphological and cultural characteristics, biochemical properties and serotyping, if required in specific cases.

\section{Antimicrobial Susceptibility Testing}

AST was carried out on the isolates by using MuellerHinton agar by Kirby-Bauer disk diffusion method. Categorization of antimicrobial resistant isolates as MDR and XDR was done following the definitions of Magiorakos et al and Tamma et al. ${ }^{17,18}$

Determination of isolates exhibiting VRE, ESBL, Carbapenemase, and MRSA characteristics was done following standard procedures. $^{16}$ Inducible-macrolidelincosamide-streptogramin-B (iMLS $\left.{ }_{\mathrm{B}}\right)$ resistance in S. aureus was detected by the disk approximation method test by placing $2 \mu \mathrm{g}$ clindamycin disk and $15 \mu \mathrm{g}$ erythromycin disk $15 \mathrm{~mm}$ apart edge to edge. The evidence of characteristic flattening zone of inhibition (ZOI) around clindamycin disk adjacent to erythromycin disk, referred to as " $\mathrm{D}$ " zone, was considered to confirm that the isolate exhibited inducible clindamycin resistance. ${ }^{16}$ For the detection of vancomycin resistance, MBL, and ESBL production, respective E-test methods were also employed.

Initial screening of ESBL among Gram-negative isolates of ESKAPE pathogens was done by using cefotaxime (CTX), ceftazidime (CAZ) and ceftriaxone (CRO) $30 \mu \mathrm{g}$ discs (BD, USA). If the ZOI was $\leq 27 \mathrm{~mm}$ for CTX, $\leq 22 \mathrm{~mm}$ for $\mathrm{CAZ}$ and $\leq 25 \mathrm{~mm}$ for $\mathrm{CRO}$, then the isolates were screened as potential ESBL-producers. These isolates were further tested for ESBL production by double disk synergy test (DDST) ${ }^{19}$ Combination Disk Test (CDT) and Epsilometer Test (E-Test) method. ${ }^{16}$

\section{Detection of MBL}

\section{Screening test for MBL Detection}

Carbapenem-resistant isolates identified by the carbapenem disk diffusion method were screened for the production of MBL. ${ }^{20}$

\section{Combined Disk Diffusion Method}

Phenotypic MBL detection was done by Combined disk method where two imipenem (IPM) disks (each $10 \mu \mathrm{g}$ ), one containing 10 microliter of $0.1 \mathrm{M}(292 \mu \mathrm{g})$ anhydrous EDTA (Thermo Fisher Scientific India Pvt. Ltd) and another without EDTA were placed $25 \mathrm{~mm}$ apart (center to center). An increase in zone diameter of $>4 \mathrm{~mm}$ around the IPM-EDTA disk compared to IPM disk alone was considered positive for MBL. ${ }^{20}$

\section{E-Test Method (Epsilometer Test)}

This test uses an E-test strips in which one end consists of a stable gradient of imipenem and the other end consists of a gradient of imipenem with a constant concentration of EDTA. MBL production was inferred positive if the MIC ratio of carbapenem alone vs imipenem+EDTA MIC was $\geq 8$. The test was done according to the manufacturer's instructions (bioMérieux SA, France).

\section{Detection of Biofilm - Tube Adherence Method}

Detection of biofilm by tube adherence method was done as proposed by Christensen et al. ${ }^{21}$ Two milliliter of Brain Heart Infusion broth supplemented with $1 \%$ glucose was used to incubate one colony of the organism for 48 hours at $37^{\circ} \mathrm{C}$. The contents were then decanted and washed by phosphate buffered saline $(\mathrm{pH}$ 7.2) thoroughly and dried. Then $1 \%$ safranin was added to the tubes and gently rotated for uniform staining. The tubes were washed with distilled water and dried by placing the tubes upside down. Presence of stained material at the inner wall of the test (liquid-air interface) was ranked by two investigators as weak biofilm producer $(+)$, medium $(++)$ or strong $(+++) .{ }^{22}$

\section{Phenotypic Detection of VRE}

Enterococcus faecium isolates showing insusceptibility to vancomycin disk $(30 \mu \mathrm{g})$ were screened as VRE. Then they were subjected to vancomycin Epsilometer test according to the manufacturer's instruction (E-Test technical manual, bioMérieux SA, France; 2018). The isolates 
showing MIC $\geq 32 \mu \mathrm{g} / \mathrm{mL}$ of vancomycin were confirmed as VRE.

\section{Molecular Detection of VRE}

Phenotypically confirmed VRE isolates were processed for molecular detection as follows.

\section{DNA Extraction}

Plasmid extraction of the VRE isolates was done by the alkaline hydrolysis method as described by Sambrook and Russell. ${ }^{23}$

\section{Polymerase Chain Reaction (PCR)}

Specific primers for amplification of vanA (Forward: GGGAAAACGACAATTGC

Reverse:GTACAATGCGGCCGTTA) and vanB (Forward: ACCTACCCTGTCTTTGTGAA reverse: AATGTCTGCTGGAACGATA) were used. ${ }^{24,25}$ For vanA and $v a n B$ amplification, PCR was performed in a thermocycler as follows- initial denaturation at $95^{\circ} \mathrm{C}$ for 5 minutes, 30 cycles for denaturation, annealing and extension at $95^{\circ} \mathrm{C}$ for 30 seconds, $54^{\circ} \mathrm{C}$ for 1 minute, and $72^{\circ} \mathrm{C}$ for 1 minute, respectively. Final elongation was done at $72^{\circ} \mathrm{C}$ for 10 minutes. ${ }^{24}$

\section{Gel Electrophoresis}

After PCR, the amplicons along with loading dye (bromophenol blue) were loaded onto $1.5 \%$ agarose gel stained with ethidium bromide of concentration $0.5 \mu \mathrm{g} / \mathrm{mL}$ and run at $60 \mathrm{~V}$ for 1 hour. The DNA bands were then analyzed in the UV transilluminator.

\section{Statistical Analysis}

All the data were statistically analysed using the IBM SPSS Statistics for windows version 16.0 (IBM Corp, Armonk, NY, USA). The P-value $<0.05$ was assumed significant for analysis.

\section{Results}

\section{Culture Positivity of Specimens}

Clinical specimens which met the criteria as recommended by ASM were processed for culture and susceptibility test. After receiving specimens from sample collection site, they were immediately transported to microbiology laboratory for further processing. Out of 8756 specimens processed, 2384 (27.2\%) showed significant growth. Among 2384 positive tested samples implicating infection, 452 (18.96\%) showed infection caused by ESKAPE pathogens. The most common isolate was Staphylococcus aureus
( $\mathrm{n}=151,33.4 \%)$ followed by $K$. pneumoniae $(\mathrm{n}=149$, $33 \%)$, P. aeruginosa $(\mathrm{n}=84,18.6 \%)$, A. baumannii $(\mathrm{n}=$ $39,8.6 \%)$, Enterococcus faecium $(\mathrm{n}=25,5.5 \%)$ and Enterobacter aerogenes (K. aerogenes) $(\mathrm{n}=4,0.9 \%)$. A higher number of ESKAPE pathogens were isolated from patients admitted to medical department $(n=281)$ followed by patients from surgical department $(n=129)$ and ICU $(\mathrm{n}=42)($ Table 1).

\section{Distribution of ESKAPE Pathogens Among the Diverse Clinical Specimens}

Among the different clinical specimens processed, ESKAPE pathogens were most commonly isolated from urine specimens followed by pus, sputum and other bodily fluids (Table 1).

Considering gender, 249 (55.1\%) ESKAPE pathogens were isolated from females and 203 (44.9\%) from males. The maximum number of patients infected were of the age group $61-70$ years $(16.4 \%)$ followed by $21-30$ years of age $(14.9 \%)$.

\section{Antimicrobial Susceptibility Pattern of ESKAPE Pathogens}

The Enterococcus faecium isolates were subjected to AST with 10 different antimicrobials. High percentage of resistance $(92 \%)$ was seen against ciprofloxacin followed by gentamicin (high level) (52\%), tetracycline (48\%) and tigecycline (48\%), vancomycin $(20 \%)$ and teicoplanin (12\%). However, all the isolates were susceptible to linezolid (Table 2).

Seventy-five percent of non-urinary isolates of S. aureus were resistant to erythromycin. A large number of $S$. aureus isolates were resistant to trimethoprimsulfamethoxazole (55\%), and ciprofloxacin (58.3\%). Almost $58 \%$ of the isolates were MRSA and $68.2 \%$ were MDR. None of the S. aureus isolates were resistant to vancomycin, teicoplanin, tigecycline and linezolid (Table 2).

Forty-nine percent of $K$. pneumoniae isolates were resistant to cefixime. It should be noted that $17.4 \%$ of the isolates showed resistance to meropenem. Approximately, $65 \%$ of urinary isolates were resistant to nitrofurantoin (Table 3).

A. baumannii showed $64 \%$ resistance to ceftazidime and cotrimoxazole each, $59 \%$ to cefepime and more than $50 \%$ to fluoroquinolones. However, they were most susceptible to ampicillin-sulbactam (64.1\%) followed by 
Table I Distribution of ESKAPE pathogens in Various Specimens

\begin{tabular}{|c|c|c|c|c|c|c|c|c|c|}
\hline Specimens & Wards & $\begin{array}{l}\text { E. faecium } \\
\text { (N) }\end{array}$ & $\begin{array}{l}\text { S. aureus } \\
\text { (N) }\end{array}$ & $\begin{array}{c}\text { K. pneumoniae } \\
(\mathbf{N})\end{array}$ & $\begin{array}{c}\text { Acinetobacter } \\
\text { baumannii } \\
\text { (N) }\end{array}$ & $\begin{array}{l}\text { P. aeruginosa } \\
\text { (N) }\end{array}$ & $\begin{array}{l}\text { E. aerogenes } \\
\text { (N) }\end{array}$ & $\begin{array}{l}\text { Total } \\
\text { (N) }\end{array}$ & Percentage \\
\hline Urine & $\begin{array}{l}\text { Medical } \\
\text { Surgical } \\
\text { ICU }\end{array}$ & $\begin{array}{l}16 \\
4 \\
3\end{array}$ & $\begin{array}{c}46 \\
11 \\
3\end{array}$ & $\begin{array}{c}78 \\
16 \\
7\end{array}$ & $\begin{array}{l}9 \\
- \\
-\end{array}$ & $\begin{array}{c}22 \\
2 \\
6\end{array}$ & $\begin{array}{l}2 \\
- \\
-\end{array}$ & 225 & $49.9 \%$ \\
\hline Pus & $\begin{array}{c}\text { Medical } \\
\text { Surgical } \\
\text { ICU }\end{array}$ & $\begin{array}{l}1 \\
- \\
-\end{array}$ & $\begin{array}{c}10 \\
52 \\
3\end{array}$ & $\begin{array}{c}3 \\
10 \\
-\end{array}$ & $\begin{array}{l}- \\
2 \\
-\end{array}$ & $\begin{array}{c}2 \\
17 \\
1\end{array}$ & $\begin{array}{l}0 \\
- \\
-\end{array}$ & 101 & $22.3 \%$ \\
\hline Sputum & $\begin{array}{c}\text { Medical } \\
\text { Surgical } \\
\text { ICU }\end{array}$ & $\begin{array}{l}1 \\
- \\
-\end{array}$ & $\begin{array}{l}6 \\
- \\
2\end{array}$ & $\begin{array}{c}23 \\
1 \\
5\end{array}$ & $\begin{array}{l}22 \\
- \\
3\end{array}$ & $\begin{array}{l}27 \\
- \\
5\end{array}$ & $\begin{array}{l}2 \\
- \\
-\end{array}$ & 97 & $21.5 \%$ \\
\hline Blood & $\begin{array}{c}\text { Medical } \\
\text { Surgical } \\
\text { ICU }\end{array}$ & $\begin{array}{l}0 \\
- \\
-\end{array}$ & $\begin{array}{l}3 \\
6 \\
-\end{array}$ & $\begin{array}{l}- \\
1 \\
1\end{array}$ & $\begin{array}{l}- \\
- \\
1\end{array}$ & $\begin{array}{l}0 \\
- \\
-\end{array}$ & $\begin{array}{l}0 \\
- \\
-\end{array}$ & 12 & $2.7 \%$ \\
\hline Semen & $\begin{array}{c}\text { Medical } \\
\text { Surgical } \\
\text { ICU }\end{array}$ & $\begin{array}{l}0 \\
- \\
-\end{array}$ & $\begin{array}{l}- \\
5 \\
-\end{array}$ & $\begin{array}{l}1 \\
- \\
-\end{array}$ & $\begin{array}{l}0 \\
- \\
-\end{array}$ & $\begin{array}{l}0 \\
- \\
-\end{array}$ & $\begin{array}{l}0 \\
- \\
-\end{array}$ & 6 & $1.3 \%$ \\
\hline $\begin{array}{l}\text { High vaginal } \\
\text { swab }\end{array}$ & $\begin{array}{c}\text { Medical } \\
\text { Surgical } \\
\text { ICU }\end{array}$ & $\begin{array}{l}0 \\
- \\
-\end{array}$ & $\begin{array}{l}2 \\
- \\
-\end{array}$ & $\begin{array}{l}1 \\
- \\
-\end{array}$ & $\begin{array}{l}0 \\
- \\
-\end{array}$ & $\begin{array}{l}0 \\
- \\
-\end{array}$ & $\begin{array}{l}0 \\
- \\
-\end{array}$ & 3 & $0.7 \%$ \\
\hline Wound swab & $\begin{array}{c}\text { Medical } \\
\text { Surgical } \\
\text { ICU }\end{array}$ & $\begin{array}{l}0 \\
- \\
-\end{array}$ & $\begin{array}{l}- \\
\\
-\end{array}$ & $\begin{array}{l}- \\
\text { I } \\
-\end{array}$ & $\begin{array}{l}0 \\
- \\
-\end{array}$ & $\begin{array}{l}0 \\
- \\
-\end{array}$ & $\begin{array}{l}0 \\
- \\
-\end{array}$ & 2 & $0.4 \%$ \\
\hline $\begin{array}{l}\text { Endotracheal } \\
\text { tube }\end{array}$ & $\begin{array}{c}\text { Medical } \\
\text { Surgical } \\
\text { ICU }\end{array}$ & $\begin{array}{l}0 \\
- \\
-\end{array}$ & $\begin{array}{l}0 \\
- \\
-\end{array}$ & $\begin{array}{l}0 \\
- \\
-\end{array}$ & $\begin{array}{l}- \\
- \\
\end{array}$ & $\begin{array}{l}- \\
- \\
\end{array}$ & $\begin{array}{l}0 \\
- \\
-\end{array}$ & 2 & $0.4 \%$ \\
\hline Ear swab & $\begin{array}{c}\text { Medical } \\
\text { Surgical } \\
\text { ICU }\end{array}$ & $\begin{array}{l}0 \\
- \\
-\end{array}$ & $\begin{array}{l}1 \\
- \\
-\end{array}$ & $\begin{array}{l}0 \\
- \\
-\end{array}$ & $\begin{array}{l}0 \\
- \\
-\end{array}$ & $\begin{array}{l}0 \\
- \\
-\end{array}$ & $\begin{array}{l}0 \\
- \\
-\end{array}$ & 1 & $0.2 \%$ \\
\hline $\begin{array}{l}\text { Broncho } \\
\text { alveolar lavage }\end{array}$ & $\begin{array}{c}\text { Medical } \\
\text { Surgical } \\
\text { ICU }\end{array}$ & $\begin{array}{l}0 \\
- \\
-\end{array}$ & $\begin{array}{l}0 \\
- \\
-\end{array}$ & $\begin{array}{l}1 \\
- \\
-\end{array}$ & $\begin{array}{l}0 \\
- \\
-\end{array}$ & $\begin{array}{l}0 \\
- \\
-\end{array}$ & $\begin{array}{l}0 \\
- \\
-\end{array}$ & 1 & $0.2 \%$ \\
\hline Suction tip & $\begin{array}{c}\text { Medical } \\
\text { Surgical } \\
\text { ICU }\end{array}$ & $\begin{array}{l}0 \\
- \\
-\end{array}$ & $\begin{array}{l}0 \\
- \\
-\end{array}$ & $\begin{array}{l}0 \\
- \\
-\end{array}$ & $\begin{array}{l}\text { I } \\
- \\
-\end{array}$ & $\begin{array}{l}- \\
- \\
-\end{array}$ & $\begin{array}{l}0 \\
- \\
-\end{array}$ & 1 & $0.2 \%$ \\
\hline Oral swab & $\begin{array}{c}\text { Medical } \\
\text { Surgical } \\
\text { ICU }\end{array}$ & $\begin{array}{l}0 \\
- \\
-\end{array}$ & $\begin{array}{l}0 \\
- \\
-\end{array}$ & $\begin{array}{l}0 \\
- \\
-\end{array}$ & $\begin{array}{l}0 \\
- \\
-\end{array}$ & $\begin{array}{l}1 \\
- \\
-\end{array}$ & $\begin{array}{l}0 \\
- \\
-\end{array}$ & 1 & $0.2 \%$ \\
\hline Total & & 25 & 151 & 149 & 39 & 84 & 4 & 452 & $100 \%$ \\
\hline
\end{tabular}

piperacillin-tazobactam (53.8\%). (Table 3). Almost 49\% of $P$. aeruginosa were resistant to ciprofloxacin, $29 \%$ to ceftazidime and $28 \%$ to cefepime. However, the majority were susceptible to piperacillin-tazobactam $(91.7 \%)$ and meropenem (84.5\%) (Table 3). All isolates of Enterobacter aerogenes ( $K$. aerogenes) were resistant to cefixime and $50 \%$ were resistant to cotrimoxazole.
Among the Gram-positive ESKAPE pathogens, more than $68 \%(n=122)$ were MDR and almost $4 \%(n=6)$ were XDR (Table 4). Nearly $27 \%(n=74)$ of the Gram-negative isolates were found to be MDR and 14\% $(n=37)$ XDR. The major drug-resistant pathogens among Gram-negative members of ESKAPE was A. baumannii (MDR and XDR $30.7 \%$ each) followed by K. pneumoniae (MDR 32.2\%, 
Table 2 Antimicrobial Resistance Pattern of Gram-Positive Isolates of ESKAPE Pathogens

\begin{tabular}{|l|l|l|l|l|}
\hline \multirow{2}{*}{ Antimicrobials } & \multicolumn{2}{l|}{$\begin{array}{l}\text { Enterococcus } \\
\text { faecium (N=25) }\end{array}$} & \multicolumn{2}{l|}{$\begin{array}{l}\text { Staphylococcus } \\
\text { aureus (N=15 })\end{array}$} \\
\cline { 2 - 5 } & N & $\%$ & N & $\%$ \\
\hline Linezolid & 0 & 0 & 0 & 0 \\
Teicoplanin & 3 & 12 & 0 & 0 \\
Vancomycin & 5 & 20 & 0 & 0 \\
Nitrofurantoin & 10 & 43.5 & 5 & 8.3 \\
Tetracycline & 12 & 48 & 3 & 1.32 \\
Doxycycline & - & - & 3 & 1.98 \\
Tigecycline & 12 & 48 & 0 & 0 \\
High-Level Gentamicin & 13 & 52 & - & - \\
Amikacin & - & - & 33 & 21.9 \\
Ciprofloxacin & 23 & 92 & 88 & 58.3 \\
Meropenem & 25 & 100 & - & - \\
Ampicillin & 25 & 100 & - & - \\
Chloramphenicol & - & - & 2 & 1.32 \\
Trimethoprim Sulphomethoxazole & - & - & 83 & 55 \\
Cefoxitin & - & - & 88 & 57.6 \\
Clindamycin & - & - & 51 & 60.7 \\
Erythromycin & - & - & 63 & 75 \\
\hline
\end{tabular}

Notes: Nitrofurantoin= For urine isolates only; Meropenem= Intrinsic resistance in E. faecium (tested for species identification); Vancomycin= E-test for vancomycin; Cefoxitin= Screening of MRSA; Clindamycin/Erythromycin= For other than urinary isolates.
XDR $12.8 \%$ ) and $P$. aeruginosa (MDR 14.3\% and XDR $7.1 \%$ each). In case of $E$. aerogenes (K. aerogenes), 2 out of 4 isolates were found to be MDR (Table 4).

\section{Molecular Characterization of vanA and vanB Genes Among VRE Isolates}

Molecular screening for vanA and vanB genes from genomic DNA/plasmid was amplified by PCR using vancomycin specific primers. All $5 \mathrm{VRE}$ isolates were found to carry vanA gene (Figure 1).

\section{Antimicrobial Resistant Gram-Negative Members of ESKAPE Pathogens ESBL- and MBL-Producing Gram-Negative ESKAPE Pathogens}

Eighty-three isolates of Gram-negative ESKAPE pathogens were resistant to third generation cephalosporin among which 37 isolates were ESBL positive by double disk synergy test, combination disk and E-test methods. Fifty-three isolates were resistant to carbapenem (imipenem) among which 23 were phenotypically confirmed to

Table 3 Antimicrobial Resistance Pattern of Gram-Negative Isolates of ESKAPE Pathogens

\begin{tabular}{|c|c|c|c|c|}
\hline \multirow[t]{2}{*}{ Antimicrobials } & $\begin{array}{l}\text { Klebsiella } \\
\text { pneumoniae } \\
(\mathbf{N}=\text { I49) }\end{array}$ & $\begin{array}{l}\text { Acinetobacter } \\
\text { baumannii } \\
(\mathbf{N}=39)\end{array}$ & $\begin{array}{l}\text { Pseudomonas } \\
\text { aeruginosa } \\
(\mathbf{N}=\mathbf{8 9})\end{array}$ & $\begin{array}{l}\text { Enterobacter } \\
\text { aerogenes } \\
(\mathbf{N}=4)\end{array}$ \\
\hline & $\mathbf{N} \%$ & $\mathbf{N} \%$ & $\mathbf{N} \%$ & $\mathbf{N} \%$ \\
\hline Amikacin & $37(24.8)$ & $19(48.7)$ & $15(17.9)$ & - \\
\hline Nitrofurantoin & 31 (64.58) & $0(0)$ & - & $2(100 \%)$ \\
\hline Trimethoprim-Sulfametoxazole & $50(33.6)$ & $25(64.1)$ & - & $2(50 \%)$ \\
\hline Ciprofloxacin & $48(32.2)$ & $21(53.8)$ & $43(48.31)$ & - \\
\hline Levofloxacin & - & $20(51.3)$ & - & - \\
\hline Amoxicillin-Clavulanic Acid & 61 (40.9) & - & - & - \\
\hline Ampicillin- Sulbactam & - & $14(35.9)$ & - & - \\
\hline Cefixime & $73(49)$ & - & - & $4(100 \%)$ \\
\hline Ceftazidime & - & $25(64.1)$ & $25(29.8)$ & - \\
\hline Cefepime & - & $23(59)$ & $24(28.6)$ & - \\
\hline Piperacillin Tazobactam & $32(21.5)$ & I8 (46.2) & $7(8.3)$ & - \\
\hline Chloramphenicol & $17(11.4)$ & - & - & - \\
\hline Meropenem & $26(17.4)$ & $20(5 \mid .3)$ & $13(15.5)$ & - \\
\hline Tigecycline & $7(4.7)$ & - & - & - \\
\hline Colistin & $0(0)$ & $0(0)$ & $0(0)$ & \\
\hline Polymyxin B & $0(0)$ & $0(0)$ & $0(0)$ & - \\
\hline Doxycycline & $20(13.4)$ & $19(48.7)$ & - & \\
\hline
\end{tabular}

Note: Nitrofurantoin= For urine isolates only. 
Table 4 Frequency of ESBL, MBL, MDR and XDR Among ESKAPE Pathogens

\begin{tabular}{|l|l|l|l|l|l|l|l|l|}
\hline \multirow{2}{*}{ Organism } & \multicolumn{2}{l}{ MDR } & \multicolumn{2}{l|}{ XDR } & \multicolumn{2}{l|}{ ESBL } & \multicolumn{2}{l|}{ MBL } \\
\cline { 2 - 9 } & $\mathbf{N}$ & $\%$ & $\mathbf{N}$ & $\%$ & $\mathbf{N}$ & $\%$ & $\mathbf{N}$ \\
\hline E.faecium & 20 & 80 & 5 & 20 & - & - & $\%$ \\
S.aureus & 102 & 67.5 & 1 & 0.6 & - & - & - \\
K. pneumoniae & 48 & 32.2 & 19 & 12.8 & 24 & 16.1 & - \\
A. baumannii & 12 & 30.76 & 12 & 30.76 & 4 & 10.3 & 12 & 4 \\
P. aeruginosa & 12 & 14.3 & 6 & 7.1 & 9 & 10.7 & 7 & 10.3 \\
E. aerogenes & 2 & 50 & 0 & 0 & 0 & 0 & 8.3 & 0 \\
\hline
\end{tabular}

Abbreviations: ESBL, extended-spectrum $\beta$-lactamase; MBL, Metallo- $\beta$-lactamase; MDR, multi-drug resistant; XDR, extensively-drug resistant.

be MBL by the combination disk and E-test methods. K. pneumoniae was the major ESBL- (16.1\%) and MBL(8.1\%) producer followed by $P$. aeruginosa $(10.7 \% \mathrm{ESBL}$, 8.3\% MBL) and A. baumannii (10.3\% ESBL and MBL each). No ESBL- or MBL-producers were isolated among Enterobacter aerogenes (K. aerogenes) (Table 4).

\section{Biofilm Production in ESKAPE Pathogens}

Of total 452 ESKAPE pathogens, 42.3\% $(n=191)$ were biofilm-producers. Among the Gram-negative isolates, A. baumannii (56.4\%) was the major biofilm-producer, and among Gram-positive isolates, 32\% of Enterococcus faecium were biofilm producers (Table 5).

\section{Relationship Between Biofilm and Antibiotic Resistance}

There was no statistical significance among biofilm and multidrug resistance in $A$. baumannii ( $\mathrm{p}=0.102)$, $P$. aeruginosa $(\mathrm{p}=0.732)$ and $E$. aerogenes (K. aerogenes) ( $\mathrm{p}$ value $=1.00)$. However, in the case of $K$. pneumoniae significant association between biofilm and MDR ( $\mathrm{p}$ value $=0.050$ ) was present. In case of Grampositive isolates there was no significant association

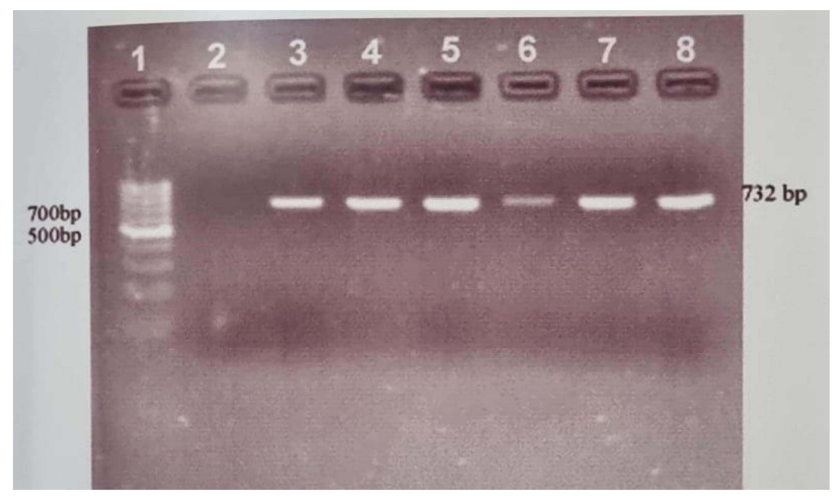

Figure I Gel Electrophoresis of PCR amplification of Van A gene. Lane I indicates DNA Ladder (I Kbp), Lane 2: Blank (Negative Control), Lane 3: Positive Control, Lane 4,5,6,7,8 VRE clinical isolate positive with van $A$ gene.
Table 5 Biofilm Producing ESKAPE Pathogens

\begin{tabular}{|c|c|c|c|c|c|}
\hline \multirow[t]{2}{*}{ Organisms } & \multicolumn{2}{|c|}{ Producers } & \multicolumn{2}{|c|}{$\begin{array}{c}\text { Non- } \\
\text { Producers }\end{array}$} & \multirow[t]{2}{*}{$\begin{array}{c}P \text { - } \\
\text { value }\end{array}$} \\
\hline & $\mathbf{N}$ & $\%$ & $\mathbf{N}$ & $\%$ & \\
\hline Enterococcus faecium & 8 & 32 & 17 & 68 & 0.484 \\
\hline Multidrug-resistant & 8 & - & 17 & - & \\
\hline Non-multidrug-resistant & 0 & - & 0 & & \\
\hline Staphylococcus aureus & 41 & 27.1 & 110 & 72.9 & 0.424 \\
\hline Multidrug-resistant & 30 & - & 73 & - & \\
\hline Non-multidrug-resistant & II & - & 37 & - & \\
\hline Klebsiella pneumoniae & 79 & 53.03 & 70 & 46.97 & 0.050 \\
\hline Multidrug-resistant & 42 & & 26 & - & \\
\hline Non-multidrug-resistant & 37 & & 44 & - & \\
\hline Acinetobacter & 22 & 56.4 & 17 & 43.6 & 0.102 \\
\hline baumannii & & & & & \\
\hline Multidrug-resistant & 16 & - & 8 & - & \\
\hline Non-multidrug-resistant & 6 & - & 9 & & \\
\hline Pseudomonas & 39 & 46.4 & 45 & 53.6 & 0.732 \\
\hline aeruginosa & & & & & \\
\hline Multidrug-resistant & 9 & - & 9 & - & \\
\hline Non-multidrug-resistant & 30 & - & 36 & - & \\
\hline Enterobacter aerogenes & 2 & 50 & 2 & 50 & 1.00 \\
\hline Multidrug-resistant & I & - & I & - & \\
\hline Non-multidrug-resistant & I & - & I & - & \\
\hline
\end{tabular}

between biofilm and multidrug resistance in S. aureus (p value $=0.424)$ and Enterococcus faecium ( $\mathrm{p}$ value = 0.484) (Table 5).

\section{Discussion}

Antimicrobial resistance is a major clinical problem worldwide in treating nosocomial and community-acquired infections caused by ESKAPE pathogens, ${ }^{26}$ and this situation is in alarming stage in Nepal as well. ${ }^{27,28}$ All members of ESKAPE pathogens fall under WHO's critical and high priority list of pathogens for research and development of 
antimicrobials, ${ }^{29}$ which further highlights the clinical importance of these organisms.

Among various clinical specimens processed, the incidence of infection with ESKAPE pathogens was found highest in urine (49.9\%) followed by pus $(22.3 \%)$ and sputum $(21.5 \%)$ specimens. In the urine specimen, major organisms isolated were $K$. pneumoniae 101 (44.8\%) followed by $S$. aureus $60(26.6 \%)$ and $P$. aeruginosa 30 (13.3\%). In the pus specimen, $S$. aureus (64.4\%) was the most frequent bacteria. This result is supported by a similar study of Pandeya et al, which was done in 164 bacteria isolated from pus specimens in a tertiary care hospital in Nepal, where the most common isolates were $S$. aureus $(32.3 \%), K$. pneumoniae $(9.1 \%)$ and $P$. aeruginosa $(6.1 \%){ }^{30}$ In the sputum specimen, the major isolates were $P$. aeruginosa (32.9\%), K. pneumoniae (29.8\%) and A. baumannii (25.7\%) which is similar to the findings of Mishra et al, which was done on 113 bacterial isolates of lower respiratory tract specimens, the major isolate being $P$. aeruginosa $(37.2 \%)$, K. pneumoniae (21.2\%) and A. baumannii (31.9\%). ${ }^{31}$

Staphylococcus aureus was the most common bacteria isolated among ESKAPE pathogens in this study. This may be because it is also a normal commensal of human skin and is capable of disseminating and causing a wide range of infections. ${ }^{32}$

In this study, $57.6 \%$ of $S$. aureus were methicillinresistant which is higher than studies conducted in two different teaching hospitals of Nepal which showed MRSA to be $45 \%$ and $39.6 \%$ respectively. ${ }^{33,34}$ The MDR $S$. aureus was $68.2 \%(\mathrm{n}=108)$ which is higher than a similar study by Sanjana et al. ${ }^{34}$ However, less than $1 \%$ of $S$. aureus were XDR. All the isolates of $S$. aureus were susceptible to vancomycin.

VRE have transferred vancomycin-resistant gene (vanA) to $S$. aureus through horizontal gene transfer containing VRE genes as transposon introducing vancomycin-resistant $S$. aureus (VRSA), ${ }^{35}$ thus compromising treatment by the last resort antibiotic against Grampositive bacteria. Five out of twenty-five (20\%) isolates of Enterococcus faecium were found vancomycin resistant in this study which is similar to the finding of Amatya et al, ${ }^{36}$ but higher than a study carried out in eastern Nepal by Acharya et al. ${ }^{37}$ In this study, five phenotypically confirmed VRE isolates (MIC $>256 \mu \mathrm{g} / \mathrm{mL}$ ) were subjected to molecular characterisation for vanA and vanB genes because VRE is predominantly mediated by these two genes in humans. ${ }^{35}$ The vanA genotype is associated with high-level resistance to both vancomycin and teicoplanin whereas vanB and vanC strains exhibit low level or variable resistance to vancomycin but they are susceptible to teicoplanin. ${ }^{38}$ However, in this study, $12 \%$ of the VRE were resistant to teicoplanin. Among the $5 \mathrm{VRE}$ isolates, 2 were susceptible to teicoplanin, while all were found to possess vanA gene. The predominance of vanA was seen similar in other studies. ${ }^{35,38,39}$ Therefore, excessive control measures should be initiated to prevent the emergence of VRE which may lead to global crisis of antimicrobials. $^{35}$

MDR among Gram-negative members of ESKAPE pathogens comprised of $K$. pneumoniae (32.2\%), which is similar to the findings by Llaca-Diaz et al. ${ }^{40}$ MDR A. baumannii were $30.7 \%$ which is higher than findings by Shrestha et al, ${ }^{11}$ and XDR were $30.7 \%$ which is similar to the findings by Llaca-Diaz et al. ${ }^{40}$ The prevalence of MDR and XDR $P$. aeruginosa was lower than the reports by Mehta and Rossolini et al. ${ }^{41,42}$ Enterobacter aerogenes (K. aerogenes) was the least prevalent isolate among the ESKAPE pathogens which was similar to the finding by Pathak et al. ${ }^{43}$

In the case of ESBL- and MBL-producers, $K$. pneumoniae was the highest ESBL- producer comprising of $16.1 \%$ which complies with the findings by Raut et $\mathrm{al} ;{ }^{44} \mathrm{MBL}$ were $8.1 \%$ which is lower than a study of Nepal et al. ${ }^{45}$ ESBL- and MBL-producing A. baumannii were $10.3 \%$ each which is similar to the results by Bhandari et al. ${ }^{46}$

Acinetobacter are largely known to produce carbapenem-inactivating OXA enzymes which also effectively hydrolyse cephalosporins. ${ }^{47}$ This study found $10.7 \%$ ESBL-producing $P$. aeruginosa and $8.3 \%$ MBLproducers which correlated with a similar study of Pathak et al. ${ }^{43}$ However, it should be noted that no phenotypic methods can reliably detect ESBL production in Acinetobacter sp. or Pseudomonas aeruginosa. Moreover, MBL detection in Acinetobacter with EDTA can result in false positive tests. ${ }^{48}$

Considering $A$. baumannii, ampicillin-sulbactam was the drug of choice with high susceptibility (64\%) excluding polymyxins. The increased susceptibility of ampicillinsulbactam against $A$. baumannii is because sulbactam containing $\beta$-lactam drug is a good therapeutic agent against A. baumannii $^{49}$ as it inhibits penicillin-binding proteins (PBPs)- PBP1 and PBP3, with a very low frequency of resistance. ${ }^{50}$ A higher percentage $(91.7 \%)$ of $P$. aeruginosa showed susceptibility against piperacillin-tazobactam 
when compared with other studies. ${ }^{41,42}$ In the case of K. pneumoniae, $82.6 \%$ were susceptible to meropenem, $78.5 \%$ piperacillin-tazobactam and $75.2 \%$ to amikacin among the first-line antibiotics. These findings were in agreement with the findings of other studies. ${ }^{51}$. There was no resistance shown by Enterobacter aerogenes (K. aerogenes) against meropenem.

Nearly $20 \%$ of Enterococcus faecium were resistant to vancomycin and $12 \%$ to teicoplanin. VRE of $5-19 \%$ was found in minced meat showing the probability of poultry and food-borne transmission in Nepal. ${ }^{52}$ Similarly, a study conducted in a hospital in Kathmandu found two VRE among nine isolates from patient's medical charts. This indicates an intense possibility of fomite-borne and foodborne transmission of MDR organisms which may lead to nosocomial infections in immuno-compromised patients. $^{53,54}$ The increasing rates of VRE in clinical samples have been observed in Europe and America too. ${ }^{35}$ In our study, linezolid was the drug of choice for VRE isolates showing $100 \%$ effectiveness in vitro.

This study showed colistin sulphate and polymyxin $\mathrm{B}$ as the effective drugs against MDR Gram-negative isolates. These drugs are regarded as reserved drugs for MDR and XDR Gram-negative bacteria. ${ }^{41,51}$ All the isolates in this study were susceptible to polymyxin; however, in Nepal resistance to polymyxin has been reported as high as $29 \%$ among Pseudomonas spp. ${ }^{55}$ These antimicrobials are used widely around the world as veterinary medicine to promote the growth of livestock/poultry in animal husbandry. ${ }^{56}$ Similarly, resistance to colistin is seen as high as $28 \%$ Escherichia coli isolates from chicken in a Nepalese study. This co-existence of MDR infection and MDR organisms (MDROs) in the food chain may exacerbate antimicrobial resistance problem leading to the emergence of PDR organisms. The U.S Food and Drug Administration (FDA) has banned the use of medically important drugs for animal growth promotion. ${ }^{57}$ Recently the health Ministry of India has also banned the manufacture, sale and distribution of colistin in poultry, aqua farming and animal feed supplements. ${ }^{58}$ It is imperative that the Nepalese government take necessary steps to ban or limit the use of broad-spectrum and strong antimicrobials in animal husbandry.

Almost $42 \%$ of ESKAPE isolates were found to be biofilm producers; however, there was no statistical significance between MDR and biofilm-producing isolates of Enterococcus faecium $(\mathrm{p}=0.484)$, S. aureus $(\mathrm{p}=0.424)$, A. baumannii $(\mathrm{p}=0.102)$, E. aerogenes $(K$. aerogenes $)(\mathrm{p}$
$=1.00)$ and $P$. aeruginosa $(\mathrm{p}=0.732)$ which concur with similar studies of Cepas et al, and Sanchez et al. ${ }^{59,60}$ However, statistical significance in between MDR $K$. pneumoniae and biofilm was seen (p 0.050). This propensity of MDR $K$. pneumoniae capable of forming biofilm was seen in a study by Vuotto et al too. ${ }^{61}$

This study provides insight into antimicrobial resistance in bacterial pathogens in Nepal. Almost 70\% (122/ 176) of the Gram-positive isolates were MDR. In the case of Gram-negative isolates, 26.8\% (74/276) and 13.4\% (37/ 276) were MDR and XDR respectively. A high level of ciprofloxacin resistance (32-54\%) in Gram-negative isolates and (58-92\%) in Gram-positive isolates was found suggesting the increase in the resistance of fluoroquinolones which is commonly used as empirical treatment for many infections.

\section{Conclusion}

This study shows a high level of antimicrobial resistance and biofilm formation among the ESKAPE isolates accounting for one of the important factors for the dissemination of antimicrobial resistance. These findings may alert clinicians when dealing with infections by ESKAPE group. Therefore, increasing antibiotic resistance is an important issue to be addressed by policymakers. Formulation of strict antibiotic stewardship policies is warranted in hospitals.

\section{Data Sharing Statement}

The data related to this study can be made available by the corresponding author if requested.

\section{Ethics and Consent to Participate}

The ethical approval for this research work was obtained from the Government of Nepal, Nepal Health Research Council (NHRC) (Reference number: 2741). Written informed consent was obtained from each patient before enrollment. For the participants under 16-years-old, consent was taken from their parent or guardian. This study was conducted in accordance with the Declaration of Helsinki.

\section{Acknowledgment}

We would like to thank Mr. Anil Kumar Shah, Research Officer, Annapurna Research Centre for the providing Molecular Laboratory facility for our research. 


\section{Author Contributions}

All authors made a significant contribution to the work reported, whether that is in the conception, study design, execution, acquisition of data, analysis and interpretation, or in all these areas; took part in drafting, revising or critically reviewing the article; gave final approval of the version to be published; have agreed on the journal to which the article has been submitted; and agree to be accountable for all aspects of the work.

\section{Funding}

This is a self-funded research done for the partial fulfillment of post graduate study.

\section{Disclosure}

The authors declare that they have no competing interest.

\section{References}

1. Coates AR, Halls G, Hu Y. Novel classes of antibiotics or more of the same? Br J Pharmacol. 2011;163(1):184-194. doi:10.1111/j.14765381.2011.01250.x

2. Blair JM, Webber MA, Baylay AJ, et al. Molecular mechanisms of antibiotic resistance. Nat Rev Microbiol. 2015;13(1):42-51. doi:10.1038/nrmicro3380

3. Molton JS, Tambyah PA, Ang BS, Ling ML, Fisher DA. The global spread of healthcare-associated multidrug-resistant bacteria: a perspective from Asia. Clin Infect Dis. 2013;56(9):1310-1318. doi:10.1093/cid/cit020

4. Levy SB, Marshall B. Antibacterial resistance worldwide: causes, challenges and responses. Nat Med. 2004;10(12):122-129. doi: $10.1038 / \mathrm{nm} 1145$

5. Kourtis AP, Hatfield K, Baggs J, et al. Emerging Infections Program MRSA Author Group: vital signs: epidemiology and recent trends in methicillin-resistant and in methicillin-susceptible Staphylococcus aureus bloodstream infections-United States. MMWR Morb Mortal Wkly Rep. 2019;68:214-219. doi:10.15585/mmwr.mm6809e1

6. Khanal LK, Jha BK. Prevalence of methicillin resistant Staphylococcus aureus (MRSA) among skin infection cases at a hospital in Chitwan, Nepal. Nepal Med College J. 2010; 12:224-228.

7. Mishra SK, Rijal BP, Pokhrel BM. Emerging threat of multidrug resistant bugs-Acinetobacter calcoaceticus baumannii complex and methicillin resistant Staphylococcus aureus. BMC Res Notes. 2013;6 (1):98-103. doi:10.1186/1756-0500-6-98

8. Mishra SK, Acharya J, Kattel HP, et al. Metallo-beta-lactamase producing gram-negative bacterial isolates. $J$ Nepal Health Res Counc. 2012;10(22):208-213.

9. Pendleton JN, Gorman SP, Gilmore BF. Clinical relevance of the ESKAPE pathogens. Expert Rev Anti Infect Ther. 2013;11:297-308. doi:10.1586/eri.13.12

10. Boucher HW, Talbot GH, Bradley JS, et al. Bad bugs, no drugs: no ESKAPE! An update from the Infectious Diseases Society of America. Clin Infect Dis. 2009;48(1):1-2. doi:10.1086/595011

11. Shrestha S, Tada T, Miyoshi-Akiyama T, et al. Molecular epidemiology of multidrug-resistant Acinetobacter baumannii isolates in a university hospital in Nepal reveals the emergence of a novel epidemic clonal lineage. Int $J$ Antimicrob Agents. 2015;46 (5):526-531. doi:10.1016/j.jiantimicag.2015.07.012
12. Browne $\mathrm{K}$, Chakraborty $\mathrm{S}$, Chen $\mathrm{R}$ et al. A new era of antibiotics: the clinical potential of antimicrobial peptides. Int J Mol Sci. 2020;21 (19):7047.

13. Mishra SK, Basukala P, Basukala O, et al. Detection of biofilm production and antibiotic resistance pattern in clinical isolates from indwelling medical devices. Curr Microbiol. 2015;70:128-134. doi:10.1007/s00284-014-0694-5

14. Praharaj I, Sujatha S, Parija SC. Phenotypic \& genotypic characterization of vancomycin resistant Enterococcus isolates from clinical specimens. Indian J Med Res. 2013;138:549-565.

15. Isenberg HD, Garcia LS. Microbiological Techniques \& MethodsLaboratory Manuals. Clinical Microbiology Procedures Handbook. 2nd ed. Washingtn DC; 2007:36-446.

16. CLSI. Performance Standards for Antimicrobial Susceptibility Testing. M100S: Wayne, Pennsylvania; 2016. 26th ed. 100-121.

17. Magiorakos A, Srinivasan A, Carey RB, et al. Multidrug-resistant, extensively drug-resistant and pandrug-resistant bacteria an international expert proposal for interim standard definitions for acquired resistance. Clin Microbiol Infect. 2012;18:268-281. doi:10.1111/ j.1469-0691.2011.03570.x

18. Tamma PD, Girdwood SC, Gopaul R, et al. The use of cefepime for treating AmpC $\beta$-lactamase-producing Enterobacteriaceae. Clin Infect Dis. 2013;57:781-788. doi:10.1093/cid/cit395

19. Garrec H, Drieux-Rouzet L, Golmard JL, et al. Comparison of nine phenotypic methods for detection of extended-spectrum $\beta$-lactamase production by enterobacteriaceae. J Clin Microbiol. 2011;49: 1048-1057. doi:10.1128/JCM.02130-10

20. Franklin C, Liolios L, Peleg AY. Phenotypic detection of carbapenem-susceptible metallo- $\beta$-lactamase- producing gram-negative bacilli in the clinical laboratory. $J$ Clin Microbiol. 2006;44:3139-3144. doi:10.1128/JCM.00879-06

21. Christensen GD, Simpson WA, Younger JJ, et al. Adherence of coagulase-negative staphylococci to plastic tissue culture plates: a quantitative model for the adherence of staphylococci to medical devices. J Clin Microbiol. 1985;22:996-1006. doi:10.1128/jcm.22. 6.996-1006.1985

22. Mathur T, Singhal S, Khan S, Upadhyay DJ, Fatma T, Rattan A. Detection of biofilm formation among the clinical isolates of staphylococci: an evaluation of three different screening methods. Indian J Med Microbiol. 2006;24:25-29. doi:10.4103/0255-0857.19890

23. Sambrook J, Russell DW. Molecular Cloning: A Laboratory Manual. New York: Cold Spring Harbor Laboratory Press; 2001.

24. Akpaka PE, Kissoon S, Jayaratne P, et al. Genetic characteristics and molecular epidemiology of vancomycin-resistant Enterococci isolates from Caribbean countries. PLoS One. 2017;12:1-11. doi:10.1371/ journal.pone. 0185920

25. Peter A, Zacharia S, Mathew EKR. Antimicrobial resistance trends with special reference to vancomycin resistance among different species of enterococci. Int J Pharma Bio Sci. 2013;4:356-363.

26. Santajit S, Indrawattana N. Mechanisms of antimicrobial resistance in ESKAPE pathogens. Biomed Res Int. 2016;2016:1-8. doi:10.1155/ 2016/2475067

27. Parajuli NP, Acharya SP, Mishra SK, et al. High burden of antimicrobial resistance among gram negative bacteria causing healthcare associated infections in a critical care unit of Nepal. Antimicrob Resist Infect Control. 2017;6:1-9. doi:10.1186/s13756-017-0222-z

28. World Health Organization. Antimicrobial resistance: global report on surveillance. World Health Organization; 2014:383-394.

29. WHO priority pathogens list for R\&D of new antibiotics; 2017. Available from: https://www.who.int/news-room/detail/27-02-2017who-publishes-list-of-bacteria-for-which-new-antibiotics-areurgently-needed. Accessed June 26, 2019.

30. Pandeya U, Raut M, Bhattarai S, Bhatt PR, Dahal PR. Bacteriological Profile and antibiogram of Bacterial Isolates from pus samples in tertiary care hospital of Kathmandu, Tribhuvan University. J Microbiol. 2018;4(1):55-62. doi:10.3126/tujm.v4i0.21678 
31. Mishra SK, Kattel HP, Acharya J, et al. Recent trend of bacterial aetiology of lower respiratory tract infection in a tertiary care centre of Nepal. Int J Infect Microbiol. 2012;1:3-8. doi:10.3126/ijim. v1i1.6639

32. Ansari S, Nepal HP, Gautam R, et al. Childhood septicemia in Nepal: documenting the bacterial etiology and its susceptibility to antibiotics. Int J Microbiol. 2014:1-6. doi:10.1155/2014/452648.

33. Parajuli NP, Parajuli H, Pandit R, Shakya J, Khanal PR. Evaluating the trends of bloodstream infections among pediatric and adult patients at a teaching hospital of Kathmandu, Nepal: role of drug resistant pathogens. Can J Infect Dis Med Microbiol. 2017;1-10. doi: $10.1155 / 2017 / 8763135$

34. Sanjana RK, Shah R, Chaudhary N, Singh YI. Prevalence and antimicrobial susceptibility pattern of methicillin-resistant Staphylococcus aureus (MRSA) in CMS-teaching hospital: a preliminary report. $J$ Coll Med Sci Nepal. 2010;6:1-6. doi:10.3126/jcmsn.v6i1.3595

35. Willems RJ, Top J, Marga van Santen D, et al. Global spread of vancomycin-resistant Enterococcus faecium from distinct nosocomial genetic complex. Emerg Infect Dis. 2005;11:821-828. doi:10.3201/ eid1106.041204

36. Amatya R, Jha B, Shrestha S, Adhikari RP, Timsina S. Prevalence of high level gentamicin and vancomycin resistance among clinical isolates of enterococci from a tertiary care hospital in central Nepal. Nepal Med College J. 2014;16:125-127.

37. Acharya A, Khanal A, Kanungo R, Mohapatra T. Characterization and susceptibility patterns of clinically important Enterococcus species in eastern Nepal. Nepal Med College J. 2007;9:250-254.

38. Miele A, Bandera M, Goldstein BP. Use of primers selective for vancomycin resistance genes to determine van genotype in enterococci and to study gene organization in VanA isolates. Antimicrob Agents Chemother. 1995;39:1772-1778. doi:10.1128/AAC.39.8.1772

39. Ko KS, Baek JY, Lee J, et al. Molecular characterization of vancomycin-resistant Enterococcus faecium Isolates from Korea Molecular characterization of vancomycin-resistant Enterococcus faecium Isolates from Korea. J Clin Microbiol. 2005;43:2303-2306. doi:10.1128/JCM.43.5.2303-2306.2005

40. Llaca-Díaz JM, Mendoza-Olazarán S, Camacho-Ortiz A, Flores S, Garza-González E. One-year surveillance of eskape pathogens in an intensive care unit of monterrey, Mexico. Chemotherapy. 2012;58:475-481. doi:10.1159/000346352

41. Mehta A, Rosenthal VD, Mehta Y, et al. Device-associated nosocomial infection rates in intensive care units of seven Indian cities. Findings of the International Nosocomial Infection Control Consortium (INICC). J Hosp Infect. 2007;67:168-174. doi:10.1016/ j.jhin.2007.07.008

42. Rossolini GM, Mantengoli E. Treatment and control of severe infections caused by multiresistant Pseudomonas aeruginosa. Clin Microbiol Infect. 2005;11:17-32. doi:10.1111/j.1469-0691.2005.01161.x

43. Pathak P, Jaishi N, Yadav BK, Shah PK. Prevalence of Extended Spectrum Beta Lactamases (ESBL) and Metallo Beta Lactamases (MBL) mediated resistance in gram negative bacterial pathogens. Tribhuvan Univ J Microbiol. 2017;4(1):49-54. doi:10.3126/tujm. v4i0.21677

44. Raut S, Gokhale S, Adhikari B. Prevalence of Extended Spectrum Beta-Lactamases among Escherichia coli and Klebsiella spp isolates in Manipal Teaching Hospital, Pokhara, Nepal. J Microbiol Infect Dis. 2015;5(2):69-75. doi:10.5799/ahinjs.02.2015.02.0179

45. Nepal K, Pant ND, Neupane B, Belbase A, Baidhya R, Shrestha RK. Extended spectrum beta-lactamase and metallo beta-lactamase production among Escherichia coli and Klebsiella pneumoniae isolated from different clinical samples in a tertiary care hospital in Kathmandu, Nepal. Ann Clin Microbiol Antimicrob. 2017;16:1-7. doi:10.1186/s12941-017-0236-7
46. Bhandari P, Thapa G, Pokhrel BM, Bhatta DR, Devkota U. Nosocomial isolates and their drug resistant pattern in ICU patients at national institute of neurological and allied sciences, Nepal. Int J Microbiol. 2015;1:1-6. doi:10.1155/2015/572163

47. Evans B, Amyes S. Oxa ß-lactamase. Clin Microbiol Rev. 2014;27:241-263. doi:10.1128/CMR.00117-13

48. Bedenić B, Ladavac R, Vranić-Ladavac M, et al. False positive phenotypic detection of metallo-beta-lactamases in Acinetobacter baumannii. Acta clinica Croatica. 2019;58:113-118. doi:10.20471/ acc.2019.58.01.15

49. Chu H, Zhao L, Wang M, Liu Y, Gui T, Zhang J. Sulbactam-based therapy for Acinetobacter baumannii infection: a systematic review and meta-analysis. Braz J Infect Dis. 2013;17:389-394. doi:10.1016/ j.bjid.2012.10.029

50. Penwell WF, Shapiro AB, Giacobbe RA, et al. Molecular mechanisms of sulbactam antibacterial activity and resistance determinants in Acinetobacter baumannii. Antimicrob Agents Chemother. 2015;59:1680-1689. doi:10.1128/AAC.04808-14

51. Shashwati N, Kiran T, Dhanvijay A. Study of extended spectrum $\beta$ lactamase producing Enterobacteriaceae and antibiotic coresistance in a tertiary care teaching hospital. J Nat Sci Biol Med. 2014;5:30. doi:10.4103/0976-9668.127280

52. Ghimire S, Basnet HB, Joshi LR, Sapkota M. Prevalence of Vancomycin resistant enterococci species in minced buffalo meat of Chitwan, Nepal. Int J Appl Sci Biotechnol. 2014;2:409-412. doi:10.3126/ijasbt.v2i4.11123

53. Nepal HP, Khanal B, Sharma SK, Gyawali N, Jha PK, Paudel R. Peritonitis in a continuous ambulatory peritoneal dialysis patient by two different species of enterococci: a rare finding. Indian J Nephrol. 2014;24:324. doi:10.4103/0971-4065.133030

54. Thapa R, Pant ND, Yadav UN, Thapa E, Singh A, Pokhrel BM. Isolation of multidrug resistant bacteria from patients medical charts. J Nepal Health Res Counc. 2017;15:146-149. doi:10.33314/ jnhrc.v15i2.1053

55. Bhandari S, Banjara MR, Lekhak B, Bhatta DR, Regmi SR. Multidrug and pan-drug resistant pseudomonas aeruginosa: a challenge in post- antibiotic era. Nepal J Sci Technol. 2013;13:197-202. doi:10.3126/njst.v13i2.7736

56. Van BTP, Brower C, Gilbert M, Grenfell BT, Levin SA, Robinson TP. Global trends in antimicrobial use in food animals. Proc Natl Acad Sci. 112;5649-5654. doi:10.1073/pnas.1503141112

57. Centers for Disease Control and Prevention (CDC). Antibiotic/ Antimicrobial Resistance, food and Food animals; 2018. Available from: https://www.cdc.gov/drugresistance/food.html. Accessed June 20,2020

58. Madlen D, Stocktona B Health Ministry bans sale, distribution of antibiotic Colistin for food producing animals; 2019. Available from: https://www.thehindubusinessline.com/news/science/health-ministrybans-sale-distribution-of-antibiotic-colistin-for-food-producinganimals/article28627093.ece. Accessed July 21, 2019.

59. Cepas V, López Y, Munoz E, et al. Relationship between biofilm formation and antimicrobial resistance in gram-negative bacteria. Microbial Drug Resist. 2019;25:72-79. doi:10.1089/mdr.2018.0027

60. Sanchez CJ, Mende K, Beckius ML, Akers KS, Romano DR, Wenke JC. Biofilm formation by clinical isolates and the implications in chronic infections. BMC Infect Dis. 2013;13:1-12. doi:10.1186/ 1471-2334-13-47

61. Vuotto C, Longo F, Balice M, Donelli G, Varaldo P. Antibiotic resistance related to biofilm formation in Klebsiella pneumoniae. Pathogens. 2014;3:743-758. doi:10.3390/pathogens3030743 


\section{Publish your work in this journal}

Infection and Drug Resistance is an international, peer-reviewed openaccess journal that focuses on the optimal treatment of infection (bacterial, fungal and viral) and the development and institution of preventive strategies to minimize the development and spread of resistance. The journal is specifically concerned with the epidemiology of antibiotic resistance and the mechanisms of resistance development and diffusion in both hospitals and the community. The manuscript management system is completely online and includes a very quick and fair peerreview system, which is all easy to use. Visit http://www.dovepress.com/ testimonials.php to read real quotes from published authors.

Submit your manuscript here: https://www.dovepress.com/infection-and-drug-resistance-journal 